\title{
Reliability testing of oxaliplatin-associated neurotoxicity questionnaire (OANQ), a pilot study
}

E. Gustafsson, Emma Litström, Carina Berterö and Jenny Drott

\section{Linköping University Post Print}

\section{Tweet}

N.B.: When citing this work, cite the original article.

The original publication is available at www.springerlink.com:

E. Gustafsson, Emma Litström, Carina Berterö and Jenny Drott, Reliability testing of oxaliplatin-associated neurotoxicity questionnaire (OANQ), a pilot study, 2015, Supportive Care in Cancer.

http://dx.doi.org/10.1007/s00520-015-2838-y

Copyright: Springer Verlag (Germany)

http://www.springerlink.com/?MUD=MP

Postprint available at: Linköping University Electronic Press

http://urn.kb.se/resolve?urn=urn:nbn:se:liu:diva-121518 
Reliability testing of Oxaliplatin-Associated Neurotoxicity Questionnaire (OANQ), a pilot study

E. Gustafsson, RN, MScN, Master student ${ }^{1}$

E. Litström, RN, MScN, Master student ${ }^{1}$

C. Berterö, RNT, PhD, Professor ${ }^{2}$

J. Drott, RN, MScN, PhD-student ${ }^{1,2}$

${ }^{1}$ Department of Surgery and Department of Medical and Health Sciences, Linköping University, Linköping, Sweden

${ }^{2}$ Division of Nursing Science, Department of Medical and Health Sciences, Linköping University, Sweden

Corresponding author:

Jenny Drott

Department of Medical and Health Sciences

Linköping University 58185 Linköping, Sweden. Tel: +46 101037061

E-mail: Jenny.Drott@liu.se 


\section{Introduction}

A common treatment in advanced colorectal cancer (CRC) patients is a combination of different

chemotherapeutic agents. Oxaliplatin is one of these drugs and it is frequently used today, usually combined with 5-fluorouracil (5-FU) and folinic acid (leucovorin) (1-2). Survival among patients with CRC has been shown to improve when oxaliplatin is added to the treatment, even when the patient is in later stages of the disease when diagnosed (3).

Oxaliplatin is known to be highly neurotoxic (4-5) and neurotoxic symptoms occur in the majority of patients treated with oxaliplatin (6). Severe neurotoxic symptoms affect the patient's daily life in many ways, and are associated with depression and affected quality of life (4). It is known that the grade of neurotoxicity is strongly dose-related (7).

There are a number of studies that have shown that the neurotoxic side effects can be permanent and that the patients can struggle for many years after the treatment is finished (7-9). It is also known, in comparison with other combinations, that patients receiving a combination that includes oxaliplatin have their symptoms for a longer time than patients receiving other combinations (5). Oxaliplatin has recently been introduced in the treatment of other forms of cancer in the gastro-intestinal tract (GI-tract), such as oesophagus-, gastric- and pancreatic cancer $(2,10-11)$.

Several studies have investigated different strategies to eliminate or reduce the neurotoxic symptoms (1112). Following that, ASCO guidelines states that there are no excising agents to prevent CIPN (13). For managing existing CIPN Duloxetine has showed positive effects on CIPN pain but also decreased numbness and tingling symptoms (13-14).

\section{Identifying risk patients}

It is of great importance to identify the patients who are at risk of developing high-grade neurotoxicity (2). Identification can eliminate the risk of developing chronic neurotoxic symptoms with functional impairment (12). Nurses have a key role to provide information to patients and also to help them identify and report early signs of neurotoxicity (1). Health professionals require valid assessments to capture early signs of neurotoxicity during and after oxaliplatin treatment (1).

In cancer care, mobile phones are becoming more widely used in the assessment, monitoring and management of symptoms (15-17). Cancer outpatients have positive experiences of reporting chemotherapyrelated symptoms using a mobile phone (16). These experiences include improvements in the management of their symptoms and better communications with health care professionals, and patients also felt more secure when their symptoms were monitored (16). Nurses have also experienced benefits since this new technology allows them to provide early interventions to patients during chemotherapy treatment (18).

There are several established assessment scales to grade and report Chemotherapy-Induced Peripheral Neuropathy (CIPN), which is an umbrella term (19). The designs of the CIPN assessments vary and they are sometimes grouped according to design, such as objective scales of common toxicity criteria (CTC), selfreported assessment scales and drug-specific assessment scales (19). The different regimes of chemotherapy present a challenge to the development of assessment scales detecting symptoms of CIPN. Oxaliplatin treatment has shown specific side-effects which highlight the need for an oxaliplatin-specific assessment. The Functional Assessment of Cancer Therapy/Gynaecologic Oncology group, Neurotoxicity (FACT/GOG-Ntx) has been 
developed to better capture oxaliplatin-specific symptoms by adding one oxaliplatin-specific item to the scale (20). Leonard et al. (21) pointed out the need for focused questions to capture to what extent Oxaliplatin Induced Peripheral Neurotoxicity (OIPN) affects daily activities. An exploratory research questionnaire was developed to capture the variety and locations of symptoms that patients undergoing oxaliplatin treatment were reporting. The questionnaire was intended to better quantify the drug-specific cold-related neuropathy symptoms, both acute and persistent, that are typical for oxaliplatin (21, Personal communication, 2014).

\section{Description of the Oxaliplatin-Associated Neurotoxicity Questionnaire (OANQ)}

The questionnaire was not given an official name by the authors (21), and therefore it has different names in various clinical trials $(19,22-23)$. In this study we decided to name the questionnaire the Oxaliplatin-Associated Neurotoxicity Questionnaire (OANQ).

The OANQ is divided into three sections corresponding to the side-effect/symptom locations: upper extremities, lower extremities, and the face/mouth area. The first part of the assessment establishes whether the patient has experienced the specific symptoms, yes or no. If the answer is yes the patient grades the severity of the symptom - hardly any to very much. Then the symptom is graded on how it affects the patient's daily activities - hardly at all bothered to extremely bothered in a functional sense. The scale ranges from 1 to 5 with a higher score indicating greater severity of the symptom and affect of daily activities. The original OANQ is in English (21) and was translated to Swedish and adjusted to Swedish settings (24). The translation of the OANQ to Swedish and back translation to English was conducted in three steps (25-26). Before the instrument was translated and adapted, the research team obtained permission from the authors of the instrument.

\section{Validity of the OANQ}

The OANQ was constructed from observational studies, experiences in clinical practice, and interviews with patients undergoing chemotherapy treatment with oxaliplatin (21; Personal communication, 2014). The mentioned method used to elaborate the questionnaire indicates strong content validity (27). In 2013, a predetermined protocol influenced by the Swedish version of the OANQ was used in a retrospective study of patients with colorectal cancer treated with oxaliplatin-based adjuvant chemotherapy (24). The study aimed to investigate the presence of documented neurotoxic side effects in the patients' medical records. Free descriptions of the physician's estimation of the patient's neurotoxic symptoms in the medical records were analysed. All symptoms in the predetermined protocol influenced by the Swedish version of the OANQ were found in the medical records except "heat sensitivity" (24). This finding also strengthens the content validity of the questionnaire (27). A formal validity of the OANQ was evaluated by a comparative analysis with the valid FACT/GOG-Ntx assessment scale (22). Pearson's correlation analysis was carried out and showed a significantly negative correlation between the FACT/GOG and the OANQ $(-0.73 ; \mathrm{p}<0.001)$ and the Ntx-subscale and the OANQ $(-0.84 ; \mathrm{p}<0.001)$ indicating a significant negative impact of patient-reported neuropathy symptoms on daily activities and quality of life. That showed that both instruments assessed a similar construct. The trial suggested further studies to evaluate the validity and reliability of the OANQ (22). A need for reliability testing was revealed in a larger research project, which the present study is a part of. Therefore, the aim of this pilot study was to test the reliability of the Swedish version of the OANQ administered throughout a selfreported mobile phone-based system. 


\section{Patients and Methods}

\section{Patients}

The participants were selected through purposive sampling between autumn 2013 to autumn 2014. The participants were admitted from four oncology clinics; two university hospitals and two regional hospitals in the south of Sweden. A patient was eligible to participate if he/she fulfilled the following criteria; had ongoing treatment with oxaliplatin, was at least eighteen years of age, able to read and write Swedish, was physically and psychologically fit to participate, and provided written informed consent. Exclusion criteria's was patients who had diabetes, recognized neurotoxicity and reduced cognitive function. Also patients who had medications that could potentially cause peripheral neurotoxicity or had previously received any neurotoxic chemotherapy. The participants also required a mobile phone with access to the Internet and the capability to use the mobile phonebased system. Of all the patients asked, twenty-four participants were included and twenty-three participants performed the test-retest. One participant was excluded due to drop-out. Many patients declined participation because of their general condition. The participants received both verbal and written information before they were admitted to the study. The participation was voluntary and the patients were able to end their participation at any time. Confidentiality was guaranteed throughout the study and informed consent to participate was written. Ethical approval was obtained from the Regional Ethical Review Board (record no: 2012/301-31).

\section{Measurements}

A test-retest of the Swedish mobile phone version of the OANQ was performed to evaluate the reliability of the questionnaire. The research group also modified and adjusted the Swedish version of the OANQ to fit a mobilesystem using Circadian Questions (CQ) (C, 21:st Century Mobile AB (http://www.cqmobil.se). In the adjustment process some of the questions were condensed to fit the mobile display but this did not interfere with the content of the questionnaire. Example: difficulty identifying objects in your hand (i.e. coin)/ difficulty identifying i.e. a coin in your hand

The time interval between the two measures was predetermined to be one hour. The date and time of the test-retest was arranged in advance to make sure that the participants, if necessary, were able to get assistance with technical issues by CQ staff monitoring the answers. The patients were instructed to answer the questionnaire as it applied to the present time, and not retrospectively. The answers from patients were directly sent via their mobile phone platform to the CQ database and were immediately accessible by the research team.

\section{Statistical Analysis}

The test-retest reliability of the OANQ was assessed by paired samples t-test, intraclass correlation (ICC) and weighted kappa. Cronbach's alpha was used to address the internal consistency. ICC is used for replicating measures from the same subject to estimate reproducibility. The ICC ranges between 0 to 1 , where $\mathrm{p}<0.4$ indicates poor reproducibility, $0.4 \leq \mathrm{p}<0.75$ indicates fair to good reproducibility and $\mathrm{p}>0.75$ indicates excellent reproducibility (28). The weighted kappa is also used to estimate reproducibility by quantifying the concordance of the same subject for repeated measures (28). The weighted kappa ranges between 0 to 1 and the kappa value is interpreted as follows; $p<0$ less than chance agreement, $0.01 \leq \mathrm{p}<0.20$ slight agreement, $0.21 \leq \mathrm{p}<0.40$ fair agreement, $0.41 \leq \mathrm{p}<0.60$ moderate agreement, $0.61 \leq \mathrm{p}<0.80$ substantial agreement and $0.81 \leq \mathrm{p}<0.99$ almost 
perfect agreement (29). For the paired samples t-test $\mathrm{p}<0.05$ was considered significant. The statistical software STATA 12.1 (StataCorp, college station, TX, USA) was used to calculate the weighted kappa. SPSS, the statistical package version 20 (SPSS Inc., Chicago, IL, USA) for Mac, was used to assess the Cronbach's Alpha, paired samples t-test and ICC.

\section{Results}

Twenty-three patients completed the test-retest, and were therefore eligible for analysis. The data contains nine $(0.7 \%)$ missing answers distributed on different items. Due to the small number of missing values no imputations were made.

\section{Patient characteristics}

The demographics and characteristics of the participants are presented in table 1 . The distribution between male and female was almost equal and the majority of the patients were diagnosed with CRC.

Please insert TABLE 1 about here

\section{Test-retest}

Weighted kappa statistics and ICC statistics for each item are presented in table 2. ICC for symptom-items: $69 \%$ indicates excellent reproducibility, $24 \%$ fair to good reproducibility and $7 \%$ poor reproducibility. ICC for affect on daily activities-items: $83 \%$ indicates excellent reproducibility, $10 \%$ fair to good reproducibility and $7 \%$ poor reproducibility. Weighted kappa for symptom-items: $59 \%$ almost perfect agreement, $28 \%$ substantial agreement, $10 \%$ moderate agreement and $3 \%$ fair agreement. Weighted kappa for affect on daily activitiesitems: $52 \%$ almost perfect agreement, $38 \%$ substantial agreement, $7 \%$ moderate agreement and $3 \%$ fair agreement.

Please insert TABLE 2 about here

Table 3 presents the paired samples t-test for the three domains of the two tests. No significant differences were found.

Please insert TABLE 3 about here

\section{Internal consistency}

The standardized Cronbach's coefficient (Cronbach's alpha) was calculated to evaluate the internal consistency of the 29-item OANQ and its domains. These figures are presented for each domain and in total in table 4. Internal consistency was good, indicating that the domains as well as the scale in total had good reliability. 


\section{Discussion}

The need for an OIPN-specific assessment to address the exact symptom type and location has been highlighted in previous studies. The statistical analysis shows overall strong reliability for the OANQ, and indicates that the questionnaire can be useful for monitoring and following up on OIPN symptoms.

The internal consistency was strong $(\alpha>0.840)$ for each symptom domain $(n=9-10)$. Cronbach's alpha for all 29 items was $\alpha>0.9$, and corresponded with the well-known problem with multi-item scales and the calculation of internal consistency. Cronbach's alpha can easily increase just by adding items (30), since the number of items is squared in the equation (31). Since some of the symptoms can occur in different areas/locations of the body, some items reappear in the three domains. The authors therefore consider the Cronbach's alpha for each symptom domain to be the main focus. The test-retest statistics for each item revealed that two items, "Difficulty feeling hot things?" and "Burning pain or discomfort without cold?", both in the upper extremity, showed poor reproducibility compared to the other items of the scale. This result could be explained by the small sample size, and by the fact that a large variation in one participant's answer would therefore affect the ICC and weighted kappa in general. Concerning the missing items, they were distributed over different items and would therefore not seem to be consistent.

Purposive sampling is suitable for evaluation and effective presentation of newly developed instruments, since the sample is selected from the researchers' knowledge of the population. To make generalizations from a study's results a probability sampling, such as randomized sampling, is needed (32). The recruitment of cancer patients was challenging, which has also been seen and highlighted in previous studies (33-35). This group of patients are often frail, undergoing tough and demanding treatment regimens and also undergoing the psychological trauma of a potential life threat. To be part of a study in this difficult period of life can be overwhelming. This was also seen in present study, since many of the patients did not meet the inclusion criteria "to be physically and psychologically fit to participate".

When it comes to sample size, there is a lack of golden standard. The sample size in our study $(n=23)$ may seem relatively small in relation to the large number of patients receiving oxaliplatin for different malignancies. Focusing too much on sample size creates great challenges for researchers of uncommon diseases or studies with a small recruitment (36). It is also interesting to discuss the importance of the sample size when reliability is the main focus, as in psychometric studies, rather than different scores and how they change over time. Hobart et al. (36) calculated that reliability testing is stable in sample sizes $\geq 20$, which would be suitable in smaller studies, such as pilot studies. That study also mentioned that these results should be interpreted with caution. The different characteristics of a scale and how these would influence the stability of the reliability tests was investigated in the same research (36). The findings of stability in relation to sample size were not influenced by the number of items, the number of sub-scales or the number of response options (36). Even though the sample size in our study was small the population seemed heterogeneous according to the distribution of sex and age and the distribution of malignancies. The study-population is transmittable to clinical settings since the largest group to be given the treatment comprised colorectal cancer patients (colorectal $n=21$, cardia $n=1$, oesophagus $n=1$ ). This distribution of patients is similar to the study population in the larger research project.

The recall period for test-retest measures must correspond to the characteristics of the phenomenon of the study, and the purpose of the assessment which explains why there is no single recall period that is best for all 
phenomena $(30,37)$. It is important to be aware of patient's possibilities to remember and also the potential risk of change in the patient's clinical status in the recall period (30). Although one hour would seem to be a short retest interval, the authors believe that it is very unlikely that the patients would remember their answers to all twenty-nine items. OIPN is a complex phenomenon in the sense that it can vary in duration and fluctuate in different environments (i.e warm/cold temperatures) (20). The time interval was estimated to one hour to make sure that the patients were stable in the interim period and to minimize the chance of a real change in the construct. It is not unlikely that the poor reproducibility of the two items in the test-retest, was caused by a true change in the symptom experience.

A majority of the patients receiving oxaliplatin suffer from neurotoxic symptoms, and yet there is still no well-accepted and effective treatment (38). This fact highlights the importance of a valid assessment to identify patients at risk. As been revealed in previous studies (39-40), the symptoms are often minimized by the patients and also by health professionals. One explanation could be the lack of valid instruments to evaluate and asses the OIPN symptoms, which could create a barrier in the communication between the health professionals and the patients. This further highlight the great need for an instrument for use in clinical practice. This would allow the health professionals to guide the patients in managing the symptoms and to carry out follow-ups continuously, during and after treatment. The follow-ups are especially important since it is known that these patients have an increased risk of developing chronic neurotoxic symptoms (41). It has also been revealed in previous studies that the use of a self-reporting instrument has positive effects on the conversation between patients and health professionals (42). The patients also experience an increased interest from the health professionals, and this makes it easier for them to talk about the symptoms (42).

Distributing a self-reported instrument through a mobile phone based platform could have benefits, as increased usefulness and accessibility, regarding both symptom reporting and follow-ups. There is only limited research on using technology in cancer follow-up, but the available research indicate that it can be valuable (4344). New technologies can have positive effects when used in the follow-up of cancer patients, such as patient empowerment, and satisfaction (43). Nurses have positive experiences of using an interactive tailored patient assessment application in the support of cancer patients since it contributes to patient-centered care (44). Further testing of the use of the mobile phone based platform in the present study will be published elsewhere as a part of the bigger research project.

Our study has limitations and strengths. First, the sample size is small, which could affect the interpretation of the results. Therefore, the results should be applied with caution, even though they may provide guidance. Potentially, the biggest challenge further studies will face in establishing reliability will be the recruitment of cancer patients. The sampling method in our study could be seen as a limitation, since a probability sample is necessary in order to generalize the results to the general population receiving oxaliplatin treatment. Purposive sampling was used to meet the patient characteristics and was suitable for the purpose of this pilot study. Since this is a pilot study, the authors recommend further testing of OANQ in a larger sample and with a randomized sampling method, to make the results more reliable. The reliability of the OANQ has been thoroughly addressed with well-known measurements, which strengthens the results. The main focus of this study was to evaluate the reliability of the OANQ. To further increase the usefulness of the instrument, future studies should focus on its sensitivity and specificity. 
In conclusion, the OANQ was tested and considered a reliable self-reporting assessment to capture the OIPN in patients receiving oxaliplatin. A valid and reliable assessment scale can contribute to the monitoring and follow-up of oxaliplatin treatment and may help nurses to guide patients in self-care actions. However, further reliability testing of the OANQ is needed.

\section{Acknowledgements}

The authors thank all patients for their participation in the study. We would also like to thank Gun Skire at CQ for technical support, Karl Wallin at LARC, Kristofer Årestedt at IMH for statistical support and discussions. We thank all nurses in the oncology clinics who had contributed to the recruitment of patients. We also are grateful to the Division of Nursing Science at the Department of Medical and Health Sciences for financial support.

\section{References}

1. Grenon N, Chan J. (2009) Managing toxicities associated with colorectal cancer chemotherapy and targeted therapy: A new guide for nurses. Clin Journal Oncol Nurs. 13(3):285-296 doi: 10.1188/09.CJON.285-296.

2. Velasco R, Bruna J, Briani C et al. (2014) Early predictors of oxaliplatin-induced cumulative neuropathy in colorectal cancer patients. J Neurol Neurosurg Pschiatry. 85(4):392-398 doi: 10.1136/jnnp-2013-305334.

3. André T, Boni C, Navarro M et al. (2009) Improved overall survival with oxaliplatin, flourouracil, and leucovorin as adjuvant treatment in stage II ord III colon cancer in the MOSAIC trial. J Clin Oncol. 27(19):3109-3116 doi: 10.1200/JCO.2008.20.6771.

4. Tofthagen C, Donovan KA, Morgan MA, Shibata D, Yeh Y. (2013) Oxaliplatin-induced peripheral neuropathy's effects on health-related quality of life of colorectal cancer survivors. Support Care Cancer. 21(12):3307-3313 doi: 10.1007/s00520-013-1905-5.

5. Land SR, Kopec JA, Cecchini RS et al. (2007) Neurotoxicity from oxaliplatin combined with weekly bolus fluorouracil and leucovorin as surgical adjuvant chemotherapy for stage II and III colon cancer: NSABP C-07. J Clin Oncol. 25(16):2205-2211.

6. Tofthagen C, McAllister RD, McMillan SC. (2011) Peripheral neuropathy in patients with colorectal cancer receiving oxaliplatin. Clin J Oncol Nurs. 15(2):182-188 doi: 10.1188/11.CJON.182-188.

7. Park BS, Goldstein D, Krishnan AV et al. (2013) Chemotherapy-induced peripheral neurotoxicity: A critical analysis. CA Cancer J Clin. 63(6):419-437 doi: 10.3322/caac.21204. 
8. Kidwell K, Yothers G, Ganz P et al. (2012) Long-term neurotoxicity effects of oxaliplatin added to fluorouracil and leucovorin as adjuvant therapy for colon cancer. Cancer. 118(22):5614-5622 doi: 10.1002/cncr.27593.

9. Pietrangeli A, Leandri M, Terzoli E, Jandolo B, Garufi C. (2006) Persistence of high-dose oxaliplatininduced neuropathy at long-term follow-up. Eur Neurol. 56(1):13-16.

10. Vatandoust A, Joshi R, Pittman KB et al. (2014) A descriptive study of persistent oxaliplatin-induced peripheral neuropathy in patients with colorectal cancer. Support Care Cancer. 22(2):513-518.

11. Sereno M, Gutiérrez-Gutiérrez G, Gómez-Raposo C et al. (2014) Oxaliplatin induced-neuropathy in digestive tumors. Crit Rev in Oncol Hematol. 89(1):166-178 doi: 10.1016/j.critrevonc.2013.08.009.

12. Wolf S, Barton D, Kottschade L, Grothey A, Loprinzi C. (2008) Chemotherapy-induced peripheral neuropathy: Prevention and treatment strategies. Eur J Cancer. 44(11):1507-1515 doi: 10.1016/j.ejca.2008.04.018.

13. Hershman DL, Lacchetti C, Dworkin RH, Lavoie Smith EM, Bleeker J, Cavaletti G et al. (2014) Prevention and management of chemotherapy-induced peripheral neuropathy in survivors of adult cancers: American Society of Clinical Oncology clinical practice guideline. J Clin Oncol. 32(18):194167. doi: 10.1200/JCO.2013.54.0914.

14. Smith EM, Pang H, Cirrincione C, Fleishman S, Paskett ED, Ahles T et al. (2013) Effect of duloxetine on pain, function, and quality of life among patients with chemotherapy-induced painful peripheral neuropathy: A randomized clinical trial. JAMA 309:1359-1367.

15. Larsen ME, Rowntree J, Young AM et al. (2008) Chemotherapy side-effect management using mobile phones. Conf Proc IEEE Eng Med Biol Soc. 2008:5152-5155 doi: 10.1109/IEMBS.2008.4650374.

16. McCann L, Maguire R, Miller M, Kearney N. (2009) Patients' perceptions and experiences of using a mobile phone-based advanced symptom management system (ASyMS®) to monitor and manage chemotherapy related toxicity. Eur J Cancer Care. 18(2):156-164 doi: 10.1111/j.1365-

2354.2008.00938.x.

17. Mirkovic J, Kaufman DR, Ruland CM. (2014) Supporting cancer patients in illness management: Usability evaluation of a mobile app. JMIR Mhealth Uhealth. 13;2(3):e33 doi: 10.2196/mhealth.3359. 
18. Maguire R, McCann L, Miller M, Kearney N. (2008) Nurses' perceptions and experiences of using of a mobile-phone-based Advanced Symptom Management System (AsyMS@) to monitor and manage chemotherapy-related toxicity. Eur J Oncol Nurs. 12(4):380-386 doi: 10.1016/j.ejon.2008.04.007.

19. Cavaletti G, Frigeni B, Lanzani F et al. (2010) Chemotherapy-induced peripheral neurotoxicity assessment: A critical revision of the currently available tools. Eur J Cancer. 46(3):479-494 doi: 10.1016/j.ejca.2009.12.008.

20. Kopec JA, Land SR, Cecchini RS et al. (2006) Validation of a self-reported neurotoxicity scale in patients with operable colon cancer receiving oxaliplatin. J Support Oncol. 4(8):W1-W8

21. Leonard GD, Wright MA, Quinn MG et al. (2005) Survey of oxaliplatin-associated neurotoxicity using and interview-based questionnaire in patients with metastatic colorectal cancer. BMC Cancer. 16(5):16 doi: 10.1186/1471-2407-5-116.

22. Driessen CML, Kleine-Bolt KME, Vingerhoets AJJM, Mols F, Vreugdenhil G. (2012) Assessing the impact of chemotherapy-induced peripheral neurotoxicity on the quality of life of cancer patients: the introduction of a new measure. Support Care Cancer. 20(4):877-881 doi: 10.1007/s00520-011-1336-0.

23. Cathomas R, Köberle D, Ruhstaller T et al. (2010) Heated $\left(37^{\circ} \mathrm{C}\right)$ oxaliplatin infusion in combination with capecitabine for metastatic colorectal carcinoma: can it reduce neuropathy? Support Care Cancer. 18(10):1263-1270 doi: 10.1007/s00520-009-0740-1.

24. Drott JE, Starkhammar H, Börjesson S, Berterö C. (2014) Oxaliplatin induced neurotoxicity among patients with colorectal cancer: Documentation in medical records - a pilot study. Open J Nurs. 4:265274 http://dx.doi.org/10.4236/ojn.2014.44031.

25. Brislin RW. (1970) Back-translation for cross-cultural research. J Cross-Cultur Psychol. 1(3):185-216 doi: $10.1177 / 135910457000100301$.

26. Acquadro C, Conway K, Giroudet C, Mear I. (2012) Linguistic validation manual for health outcome assessments. MAPI Institute. Lyon.

27. Mokkink LB, Terweea CB, Patrick DL et al. (2010) The COSMIN study reached international consensus on taxonomy, terminology, and definitions of measurement properties for health-related patient-reported outcomes. J Clin Epidemiol. 63(7): 737-745 doi: 10.1016/j.jclinepi.2010.02.006.

28. Rosner B. (2011) Fundamentals of biostatistics. Brooks. Boston. 
29. Viera AJ \& Garrett JM. (2005) Understanding interobserver agreement: The Kappa Statistic. Fam Med. 37(5):360-363

30. Polit DF. (2014) Getting serious about test-retest reliability: a critique of retest research and some recommendations. Qual Life Res 23(6):1713-1720 doi: 10.1007/s11136-014-0632-9.

31. Field A. (2013) Discovering statistics using IBM SPSS statistics: and sex and drugs and rock 'n' roll. Fourth edition. Sage. Los Angeles.

32. Polit DF, Beck CT. (2012) Nursing research, generating and assessing evidence for nursing practice. Ninth Edition. Lippincott Williams \& Wilkins. Philadelphia.

33. Berger A, Edwards Neumark D, Chamberlain J. (2007) Enhancing recruitment and retention in randomized controlled clinical trials of cancer symptom management. Oncol Nurs Forum. 34(2):e17e22.

34. Ganz PA, Land SR, Antonio C et al. (2009) Cancer survivorship research: the challenge of recruiting adult long term cancer survivors from a cooperative clinical trials group. J Cancer Surviv. 3(3):137-147 doi: 10.1007/s11764-009-0093-2.

35. Paul C, Courtney R, Sanson-Fisher R et al. (2014) A randomized controlled trial of the effectiveness of a pre-recruitment primer letter to increase participation in a study of colorectal screening and surveillance. BMC Med Res Methodol. 14:44 http://biomedcentral.com/1471-2288/14/44.

36. Hobart JC, Cano SJ, Warner TT, Thompson AJ. (2012) What sample sizes for reliability and validity studies in neurology? J Neurol. 259(12):2681-2694 doi:10.1007/s00415-012-6570-y.

37. Stull DE, Kline Leidy N, Parasuraman B, Chassany O. (2009) Optimal recall periods for patientreported outcomes: challenges and potential solutions. Curr Med Res Opin. 25(4):929-942 doi: $10.1185 / 03007990902774765$.

38. Seretny M, Currie GL, Sena ES et al. (2014) Incidence, prevalence, and predictors of chemotherapyinduced peripheral neuropathy: A systematic review and meta-analysis. Pain. 155(12):2461-2470 doi: http://dx.doi.org/10.1016/j.pain.2014.09.020.

39. Bakitas, M. (2007) Background noise; The experience of chemotherapy-induced peripheral neuropathy. Nurs Res. 56(5): 323-331. 
40. Bennett BK, Park SB, Lin CSY, Friedlander ML, Kiernan MC, Goldstein D. (2012) Impact of oxaliplatin-induced neuropathy: a patient perspective. Support Care Cancer. 20(11):2959-2967.

41. Beijers AJM, Mols F, Vreugdenhil G. (2014) A systematic review on chronic oxaliplatin-induced peripheral neuropathy and the relation with oxaliplatin administration. Support Care Cancer. 22(7):1999-2007 doi: 10.1007/s00520-014-2242-z.

42. Velikova G, Keding A, Harley et al. (2010) Patients report improvements in continuity of care when quality of life assessments are used routinely in oncology practice: Secondary outcomes of a randomized controlled trial. Eur J Cancer. 46(13):2381-2388 doi:10.1016/j.ejca.2010.04.030.

43. Dickinson R, Hall S, Sinclair JE, Bond C, Murchie P. (2014) Using technology to deliver cancer follow-up: a systematic review. BMC Cancer. 14(1):311 http://biomedcentral.com/1471-2407/14/311.

44. Børøsund E, Ruland CM, Moore S, Ekstedt M. (2014) Nurses' experiences of using an interactive tailored patient assessment tool one year past implementation. Int J Med Inform. 83(7):e23-e34 http://dx.doi.org/10.1016/j.ijmedinf.2013.10.010 